\title{
When Do CAPITAL INFLOW SURGES ENd In TEARS?
}

Atish R. Ghosh, Jonathan D. Ostry, and Mahvash S. Qureshi

\section{ONLINE APPENDIX}

This appendix provides information on data sources, variable definitions, and the identified surge episodes and financial crisis in the sample.

Table A.1 List of countries in the sample

\begin{tabular}{lll}
\hline Albania & Estonia & Panama \\
Algeria & Georgia & Peru \\
Argentina & Guatemala & Philippines \\
Armenia & Hungary & Poland \\
Belarus & India & Romania \\
Bosnia \& Herzegovina & Indonesia & Russian Federation \\
Brazil & Jamaica & Serbia \\
Bulgaria & Jordan & Slovak Republic \\
Chile & Kazakhstan & South Africa \\
China & Korea, Rep. & Sri Lanka \\
Colombia & Latvia & Thailand \\
Costa Rica & Lebanon & Tunisia \\
Croatia & Lithuania & Turkey \\
Czech Republic & Macedonia, FYR & Ukraine \\
Dominican Republic & Malaysia & Uruguay \\
Ecuador & Mexico & Venezuela, RB \\
Egypt, Arab Rep. & Morocco & Vietnam \\
E Salvador & Pakistan & \\
\hline
\end{tabular}

Note: Countries in the sample are emerging markets as identified by the IMF's Early Warning Exercise for Emerging Markets. 
Table A.2 List of identified surge episodes and crash endings

\begin{tabular}{|c|c|c|c|c|c|c|c|c|}
\hline Country & Episode & $\begin{array}{l}\text { Financial } \\
\text { crisis }\end{array}$ & Country & Episode & $\begin{array}{l}\text { Fina ncial } \\
\text { c risis }\end{array}$ & Country & Episode & $\begin{array}{l}\text { Financial } \\
\text { crisis }\end{array}$ \\
\hline Albania & 2006- 14 & N.a. & Estonia & $1996-97$ & No & Panama & $2010-11$ & No \\
\hline Argentina & 1993 & Yes & Estonia & 2003-07 & No & Panama & 2013- 14 & N.a. \\
\hline Argentina & 1997-98 & No & Georgia & 2005-08 & No & Peru & 1994-97 & No \\
\hline Armenia & 1996-200( & No & Georgia & 2011- 12 & No & Peru & 2002 & No \\
\hline Armenia & 2008 & No & Guatemala & 1987 & No & Peru & 2007-08 & No \\
\hline Armenia & 2013 & N.a. & Guatemala & $1991-94$ & No & Peru & $2010-13$ & N.a. \\
\hline Belarus & 1997 & Yes & Guatemala & 1997-98 & No & Philippines & 1991 & No \\
\hline Belarus & 2004 & No & Guatemala & 2000-03 & No & Philippines & 1994- 97 & Yes \\
\hline Belarus & 2007 & Yes & Hungary & 1993-95 & No & Philippines & 1999 & No \\
\hline Belarus & 2009-11 & No & Hungary & $1998-2000$ & No & Philippines & 2010 & No \\
\hline Belarus & 2013 & N.a. & Hungary & 2004-06 & Yes & Poland & 1995- 96 & No \\
\hline Bosnia & 2001 & No & Hungary & 2008 & Yes & Poland & $1998-2000$ & No \\
\hline Bosnia & 2003-05 & No & India & 2007 & No & Poland & 2005 & No \\
\hline Bosnia & 2007-08 & No & Indonesia & 2014 & N.a. & Poland & 2007-11 & No \\
\hline Brazil & $1980-81$ & Yes & Jamaica & 1992 & No & Romania & 1980 & No \\
\hline Brazil & 1994 & Yes & Jamaica & 1996 & Yes & Romania & 1997-98 & No \\
\hline Brazil & 2007 & No & Jamaica & 2001-02 & No & Romania & 2001-08 & No \\
\hline Brazil & 2014 & N.a. & Jamaica & 2004-08 & No & Russia & 2007 & Yes \\
\hline Bulgaria & $1992-93$ & No & Jamaica & 2011 & No & Serbia & 2007-08 & No \\
\hline Bulgaria & 2000-08 & No & Jamaica & 2014 & N.a. & Serbia & 2011 & No \\
\hline Chile & $1980-81$ & Yes & Jordan & 1988 & Yes & Slovak Rep. & 1996 & Yes \\
\hline Chile & $1989-90$ & No & Jordan & $1991-92$ & No & Slovak Rep. & 1998-99 & No \\
\hline Chile & 1992- 97 & No & Jordan & 2005- 11 & No & Slovak Rep. & 2002 & No \\
\hline Chile & 2011 & No & Jordan & 2013 & N.a. & Slovak Rep. & 2004-05 & No \\
\hline China & 1994 & No & Kazakhstan & $1996-97$ & Yes & Slovak Rep. & 2007 & No \\
\hline China & 2004 & No & Kazakhstan & 2001 & No & South Africa & $2006-07$ & No \\
\hline China & 2010 & No & Kazakhstan & 2003-04 & No & South Africa & 2009 & No \\
\hline Colombia & 1996-97 & Yes & Kazakhstan & 2006 & Yes & South Africa & 2012 & No \\
\hline Colombia & 2007 & No & Korea & 1980 & No & Sri Lanka & 1980 & No \\
\hline Colombia & 2013- 14 & N.a. & Korea & 2009 & No & Sri Lanka & 1982 & No \\
\hline Costa Rica & 1995 & No & Latvia & 1995 & Yes & Sri Lanka & 1993-94 & No \\
\hline Costa Rica & 1999 & No & Latvia & 1999 & No & Sri Lanka & 2009 & No \\
\hline Costa Rica & 2002 & No & Latvia & 2001 & No & Sri Lanka & 2011- 13 & N.a. \\
\hline Costa Rica & 2005-08 & No & Latvia & 2004-07 & Yes & Thailand & 1981 & Yes \\
\hline Costa Rica & 2011- 14 & N.a. & Lebanon & 2003 & No & Thailand & 1988-96 & Yes \\
\hline Croatia & $1996-97$ & Yes & Lebanon & 2008-09 & No & Thailand & 2010 & No \\
\hline Croatia & 1999 & No & Lebanon & 2014 & N.a. & Tunisia & $1981-82$ & No \\
\hline Croatia & 2001 & No & Lithuania & 1997-98 & No & Tunisia & 1984 & No \\
\hline Croatia & 2003 & No & Lithuania & 2003 & No & Tunisia & 1993 & No \\
\hline Croatia & 2006-07 & No & Lithuania & 2005- 07 & No & Tunisia & 2006 & No \\
\hline Czech Rep. & 1995- 96 & Yes & Macedonia & 2002 & No & Tunisia & 2008-09 & No \\
\hline Czech Rep. & 2000-02 & No & Macedonia & 2004-08 & No & Tunisia & 2012- 14 & N.a. \\
\hline Czech Rep. & 2004 & No & Malaysia & $1980-85$ & No & Turkey & 2004-08 & No \\
\hline Dominican Rep & $2000-01$ & Yes & Malaysia & $1991-93$ & No & Turkey & $2010-14$ & N.a. \\
\hline Dominican Rep & 2008 & No & Malaysia & 1995- 96 & Yes & Ukraine & 2005 & No \\
\hline Dominican Rep & $2010-11$ & No & Mexico & 1981 & Yes & Ukraine & 2007 & Yes \\
\hline Dominican Rep & $2013-14$ & N.a. & Mexico & $1991-93$ & Yes & Ukraine & $2012-13$ & N.a. \\
\hline Ecuador & 1990- 92 & No & Mexico & 1997 & No & Uruguay & 1980 & Yes \\
\hline Ecuador & 2002 & No & Mexico & 2013 & N.a. & Uruguay & 1982 & Yes \\
\hline Egypt & 2005 & No & Morocco & 2013- 14 & N.a. & Uruguay & 2005-08 & No \\
\hline El Salvador & 1998 & No & Pakistan & 2006- 07 & No & Uruguay & 2011- 14 & N.a. \\
\hline El Salvador & 2003 & No & Panama & 1997-99 & No & Venezuela & 1990 & No \\
\hline El Salvador & 2006 & No & Panama & 2001 & No & Vietnam & $1996-97$ & Yes \\
\hline El Salvador & 2008 & No & Panama & 2005 & No & Vietnam & 2003 & No \\
\hline El Salvador & 2012 & No & Panama & 2007-08 & No & Vietnam & 2007-09 & No \\
\hline
\end{tabular}

Source: Authors' calculations.

Note: N.a.= if no information is available on whether the country had a crisis or not. Financial crisis are identified as a banking or currency crisis (as defined in Laeven and Valencia (2013)) within two years of the end of a surge episode. 
Table A.3 Variable definitions and data sources

\begin{tabular}{|c|c|c|}
\hline Variables & Description & Source \\
\hline Bank foreign liabilities & In billions of USD & IMF's IFS database \\
\hline Capital account openness & Index (high=liberalized; low=closed) & Chinn- Ito $(2008)^{1}$ \\
\hline Commodity prices & Index & IMF's WEO database \\
\hline Current account balance & In billions of USD & IMF's WEO database \\
\hline Exchange rate regime & De facto (1=Fixed; $2=$ Intermediate; $3=$ Flexible $)$ & Ghosh et al. $(2015)^{2}$ \\
\hline External debt & In billions of USD & IMF's WEO database \\
\hline Financial crisis & $\begin{array}{l}\text { Binary variable equal to } 1 \text { for banking or currency crisis, } \\
\text { zero otherwise }\end{array}$ & Laeven and Valencia $(2013)^{3}$ \\
\hline Foreign exchange reserves & Stock (In billions of USD) & IMF's IFS database \\
\hline GDP current/constant prices & In billions of USD (or LC) & IMF's WEO database \\
\hline Institutional quality & Index (average of ICRG's 12 political risk components) & Political Risk Group \\
\hline Net capital flows & $\begin{array}{l}\text { Net financial flows excluding financing items and other } \\
\text { investment liabilities of general government (In USD bln.), } \\
\text { i.e., the difference between IFS series codes "..4995W.9" } \\
\text { and "..4753ZB9" (in terms of BPM5 presentation) }\end{array}$ & IMF's IFS database \\
\hline Money market rate & In percent & IMF's IFS database \\
\hline Private sector credit & In billions of LC & IMF's IFS database \\
\hline Real Effective Exchange Rate & Index & INS database \\
\hline Output gap & $\begin{array}{l}\text { Log difference between real GDP and real GDP trend } \\
\text { (obtained from HP filter) }\end{array}$ & Authors' calculations \\
\hline Overvaluation & $\begin{array}{l}\text { Log difference between REER and REER trend (obtained } \\
\text { from HP filter) }\end{array}$ & Authors' calculations \\
\hline Real GDP per capita & In USD & IMF's WEO database \\
\hline Real interest rate & $\begin{array}{l}{[(1+\text { nominal interest rate }) /(1+\text { expected inflation })]-1, \text { where }} \\
\text { expected inflation is one-period ahead inflation }\end{array}$ & Authors' calculations \\
\hline S\&P 500 index returns volatility & $\begin{array}{l}\text { Annual average of twelve- month rolling standard } \\
\text { deviation of S\&P } 500 \text { index annual returns }\end{array}$ & $\begin{array}{l}\text { Authors' calculations based } \\
\text { on data from Bloomberg. }\end{array}$ \\
\hline Surge & $\begin{array}{l}\text { Net capital flow/GDP (as defined above) for a country lies } \\
\text { in the top } 30 \text { th percentile of the country-specificand full } \\
\text { sample's distribution of net capital flows/GDP }\end{array}$ & $\begin{array}{l}\text { Authors' calculations based } \\
\text { on data from IMF's IFS } \\
\text { database }\end{array}$ \\
\hline U.S. 3-month Treasury Bill rate & In percent & IMF's WEO and Bloomberg \\
\hline \multicolumn{3}{|c|}{$\begin{array}{l}\text { V Chinn, M., and H. Ito, 2008, "A New M easure of Financial Openness," Journal of Comparative Policy Analysis } 10 \text { (3): } 309-322 . \\
2 / \text { Ghosh, A., J. Ostry, and M. Qureshi, 2015, "Exchange Rate M anagement and Crisis Susceptbility: A Reassessment," IMF Economic } \\
\text { Review } 63 \text { (1): 238-276. }\end{array}$} \\
\hline
\end{tabular}

